\title{
Reviewer's comment concerning "Spinal fractures in patients with ankylosing spinal disorders: a systematic review of the literature on treatment, neurological status and complications" (L. A. Westerveld et al. Ms-no: ESJO-D-08-00152R1)
}

\author{
Frank Kandziora
}

Accepted: 13 November 2008/Published online: 9 December 2008

(C) Springer-Verlag 2008

I would like to congratulate the authors to their excellent systematic review on spinal fractures in patients with ankylosing spondylitis (AS) and diffuse idiopathic skeletal hyperostosis (DISH) especially focusing on neurological outcome and complications in conservative and operative treatment. One of the most important conclusions of the manuscript is that "surgical treatment may be favourable for patients with an ankylosed spine and spinal fracture, as this treatment option may be associated with lower complication and mortality rates and may lead to neurological improvement more frequently". Although this is an extremely satisfying statement, especially for a spine surgeon, we have to ask ourselves if the methodology used in this review is adequate to support this statement.

It can be argued that comparing operative with conservative treatment of fractures in patients with ankylosing spondylitis is improper, because if somebody followed the recommendations in standard textbooks, the ideal candidate for surgery would be a patient with ankylosing spondylitis having a severe trauma leading to an unstable fracture with a neurological deficit. On the other side, the ideal indication for conservative treatment would be a minor trauma leading to a stable fracture without a neurological deficit. No prospective controlled or randomised studies are available covering that topic. Therefore, only

retrospective case series or case reports with a grade 4 level of evidence could be included in this review. Due to the lack of controlled or randomised studies, it can be assumed that the authors of the studies included in this review were following the above mentioned widely excepted indication criteria. Therefore, it might be argued that the comparison of the two treatment strategies is improper, because two different entities (stable vs. unstable fracture) with different severity (minor vs. severe trauma) were treated. In other words, it can be argued that this review compares apples and oranges.

However, this would still be very satisfactory for a spine surgeon, because if the severe injured patients (apples) treated surgically would still do better than the light injured patients (oranges) treated conservatively, this would even be a much stronger argument for a surgical treatment. Although this is very appealing, especially for a spine surgeon, at the end of the day it is once again just a grade 4 level of evidence.

Now it is our task to improve the level of evidence by performing good prospective controlled-maybe even randomised — studies by looking separately either on apples or oranges to further improve especially the surgical treatment of our patients with ankylosing spinal disorders.

This comment refers to the Review doi:10.1007/s00586-008-0764-0.

F. Kandziora $(\bowtie)$

Zentrum für Wirbelsäulenchirurgie und Neurotraumatologie,

Berufsgenossenschaftliche Unfallklinik, Friedberger Landstrasse

430, 60389 Frankfurt am Main, Germany

e-mail: frank.kandziora@bgu-frankfurt.de

URL: http://www.bgu-frankfurt.de; http://www.spinesurgery.de 